\title{
Less explored off-label indications of dexmedetomidine
}

\author{
Abhijit Nair \\ Department of Anesthesiology, Basavatarakam Indo-American Cancer Hospital and Research Institute, Banjara \\ Hills, India
}

We read with great interest the article titled "An introduction to the various roles of dexmedetomidine" by Kim [1] in the recent edition of the Korean Journal of Anesthesiology. Dexmedetomidine has emerged as an essential drug in current balanced anesthesia practice as it confers hemodynamic stability, reduces opioid consumption due to its sparing effect, facilitates early extubation as it reduces the inhalational anesthetic requirement, and works wonderfully when used along with regional anesthesia either as an intravenous infusion or as an adjunct to central neuraxial block and peripheral nerve blocks.

The drug has also been used successfully in a few other offlabel indications. Similar to midazolam, ketamine, and triclofos, dexmedetomidine has been used as an oral premedication at doses of $2-4 \mu \mathrm{g} / \mathrm{kg}$ in pediatric patients. Faritus et al. [2] used dexmedetomidine premedication at a dose of $2 \mu \mathrm{g} / \mathrm{kg}$ in pediatric patients aged 2-12 years scheduled for congenital heart disease surgery and found dexmedetomidine superior to oral midazolam premedication. When dexmedetomidine is administered orally, there is extensive first-pass metabolism with oral bioavailability of around $16 \%$. Therefore, a higher dose is required when it is used for oral premedication [3].

Intra-articular infiltration using a cocktail of agents has been explored for arthroplasty and arthroscopic surgery. Drugs like

\footnotetext{
Corresponding author: Abhijit Nair, M.D.

Department of Anesthesiology, Basavatarakam Indo-American Cancer Hospital and Research Institute, Road No. 10, Banjara Hills, Hyderabad 500034, Telangana, India.

Tel: 91-40-23551235, Fax: 91-40-23553556

Email: abhijitnair95@gmail.com

ORCID: http://orcid.org/0000-0003-2506-0301
}

Received: January 17, 2017.

Revised: January 21, 2017.

Accepted: January 22, 2017.

Korean J Anesthesiol 2017 June 70(3): 361-362

https://doi.org/10.4097/kjae.2017.70.3.361 local anesthetics (lidocaine, bupivacaine, and ropivacaine), clonidine, opioids (morphine and fentanyl), ketorolac, and magnesium have been used in various combinations and at different concentrations for intra-articular infiltration. Alipour et al. [4] randomized 46 patients scheduled for arthroscopic knee surgery and used $1 \mu \mathrm{g} / \mathrm{kg}$ of dexmedetomidine infiltration (in a volume of $25 \mathrm{ml}$ ) in one group and placebo (normal saline) in the other group. The 23 patients who received dexmedetomidine infiltration were more comfortable, with significantly less opioid consumption postoperatively.

Alpha $_{2}$ agonists inhibit the release of norepinephrine at peripherally located nociceptors by acting on alpha $a_{2}$ adrenergic presynaptic receptors. Dexmedetomidine could exert some effect due to systemic absorption, but the dose when used as an adjunct in infiltration is the same as the intravenous loading dose. The recommended dose used intra-articularly, subcutaneously, and for infiltration is the same as that used for a loading dose. The systemic absorption is definitely slower than when given intravenously.

Lidocaine with adrenaline skin infiltration is a standard practice in many surgeries, especially orthopedic, head and neck, and neurosurgery, and reduces the blood loss via vasoconstriction caused by adrenaline. Postoperative bupivacaine/ropivacaine skin infiltration is practiced at several centers. The addition of dexmedetomidine to lidocaine with adrenaline for skin infiltration has not been explored in detail. Skin infiltration with $1 \mu \mathrm{g} / \mathrm{kg}$ of dexmedetomidine has been shown to improve postoperative analgesia. Mandal et al. [5] used $1 \mu \mathrm{g} / \mathrm{kg}$ of dexmedetomidine with lidocaine and adrenaline infiltration for maxillofacial surgery preoperatively after inducing anesthesia and found that there was less surgical bleeding, reduced anesthetic and opioid requirements, stable hemodynamics, and early discharge from the postanesthesia care unit when compared with lidocaine and adrenaline plus placebo (normal saline). Bradycardia and drowsiness are problems encountered after subcutaneous use and when used in infiltration.

(c) This is an open-access article distributed under the terms of the Creative Commons Attribution Non-Commercial License (http://creativecommons.org/ licenses/by-nc/4.0/), which permits unrestricted non-commercial use, distribution, and reproduction in any medium, provided the original work is properly cited. 
Although dexmedetomidine appears to be effective in several off-label indications, adequately powered studies and subsequent meta-analyses are required before considering dexme- detomidine as a cost-effective modality for the above-mentioned situations.

\section{References}

1. Kim JK. An introduction to the various role of dexmedetomidine. Korean J Anesthesiol 2016; 69: 543-4.

2. Faritus SZ, Khazaee-Koohpar M, Ziyaeifard M, Mehrabanian MJ. Oral dexmedetomidine versus midazolam as anesthetic premedication in children undergoing congenital heart surgery. Anesth Pain Med 2015; 5: e25032.

3. Anttila M, Penttilä J, Helminen A, Vuorilehto L, Scheinin H. Bioavailability of dexmedetomidine after extravascular doses in healthy subjects. Br J Clin Pharmacol 2003; 56: 691-3.

4. Alipour M, Tabari M, Faz RF, Makhmalbaf H, Salehi M, Moosavitekye SM. Effect of dexmedetomidine on postoperative pain in knee arthroscopic surgery; a randomized controlled clinical trial. Arch Bone Jt Surg 2014; 2: 52-6.

5. Mandal D, Das A, Chhaule S, Halder PS, Paul J, RoyBasunia S, et al. The effect of dexmedetomidine added to preemptive (2\% lignocaine with adrenaline) infiltration on intraoperative hemodynamics and postoperative pain after ambulatory maxillofacial surgeries under general anesthesia. Anesth Essays Res 2016; 10: 324-31. 